\title{
EchoGéo
}

2| 2007

Itinéraires et trajectoires des migrants

\section{Une expérience d'application multimédia à un bassin-versant}

Françoise Duraffour

\section{OpenEdition}

Journals

Édition électronique

URL : https://journals.openedition.org/echogeo/1557

DOI : 10.4000/echogeo.1557

ISSN : 1963-1197

Éditeur

Pôle de recherche pour l'organisation et la diffusion de l'information géographique (CNRS UMR 8586)

Référence électronique

Françoise Duraffour, « Une expérience d'application multimédia à un bassin-versant », EchoGéo [En ligne], 2 | 2007, mis en ligne le 19 septembre 2007, consulté le 31 juillet 2021. URL : http:// journals.openedition.org/echogeo/1557 ; DOI : https://doi.org/10.4000/echogeo.1557

Ce document a été généré automatiquement le 31 juillet 2021.

EchoGéo est mis à disposition selon les termes de la licence Creative Commons Attribution - Pas d'Utilisation Commerciale - Pas de Modification 4.0 International (CC BY-NC-ND) 


\title{
Une expérience d'application multimédia à un bassin-versant
}

\author{
Françoise Duraffour
}

1 Les géographes, peut-être plus que d'autres thématiciens ont en permanence recours à l'image. Qu'il s'agisse de cartes, de films, de photographies ou d'images satellitaires, l'image est au cœur de leurs préoccupations. Elle n'est pas seulement la représentation d'un résultat, elle est le résultat ou la recherche elle-même. Plus que dans d'autres disciplines, écrit et image se travaillent mutuellement.

2 Comment dans ce contexte, ne pas être tenté de développer des applications géographiques de type multimédia? Elles mêlent étroitement les différents supports de formulation (support textuel, support graphique) tout en privilégiant l'image fixe ou animée. Le terme "multimédia» est largement galvaudé ; quelques précisions de terminologie s'imposent.

3 Une application multimédia n'est pas, selon nous, un ensemble de pages « web » reliées entre elles par les liens appelés « liens hypertextes». Elle est avant tout un logiciel (ou un ensemble de logiciels) paramétré sur mesure pour une thématique particulière. Ce logiciel intègre bien sûr différents médias graphiques et textuels; il offre surtout un ensemble de fonctions cohérentes autour d'une même problématique de recherche.

1. Vers des applications concrètes

4 Le discours autour de l'image en géographie ne suffit pas. Il appelle l'expérimentation autour de réalisations concrètes et accessibles. A ce titre, nous avons développé au sein de l'UMR Prodig un application pilote intitulée «le Suzon, les hommes et la ville de Dijon, usages, gestion et protection d'un cours d'eau à travers le temps » à laquelle ont participé plusieurs compétences différentes : photographe, informaticien et bien sûr thématicien.

5 Le projet est actuellement accessible aux membres de l'UMR. Lorsque des compléments thématiques seront apportés et les accords réglementaires relatifs à la diffusion de quelques documents obtenus, cette application pourra être diffusée via internet.

2. Une richesse fonctionnelle 
6 L'application multimédia offre de nombreuses fonctions. Un menu « interactif » permet par exemple de déplier ou de replier toute partie ou sous-partie d'un projet puis d'y accéder directement. Contrairement à l'ouvrage ou à l'article, la lecture n'est pas a priori linéaire. Les parcours de lecture sont infinis, les liens multiples. Bien qu'elle soit évidemment structurée, l'application multimédia n'impose aucun sens de lecture et laisse le lecteur libre de construire son propre parcours.
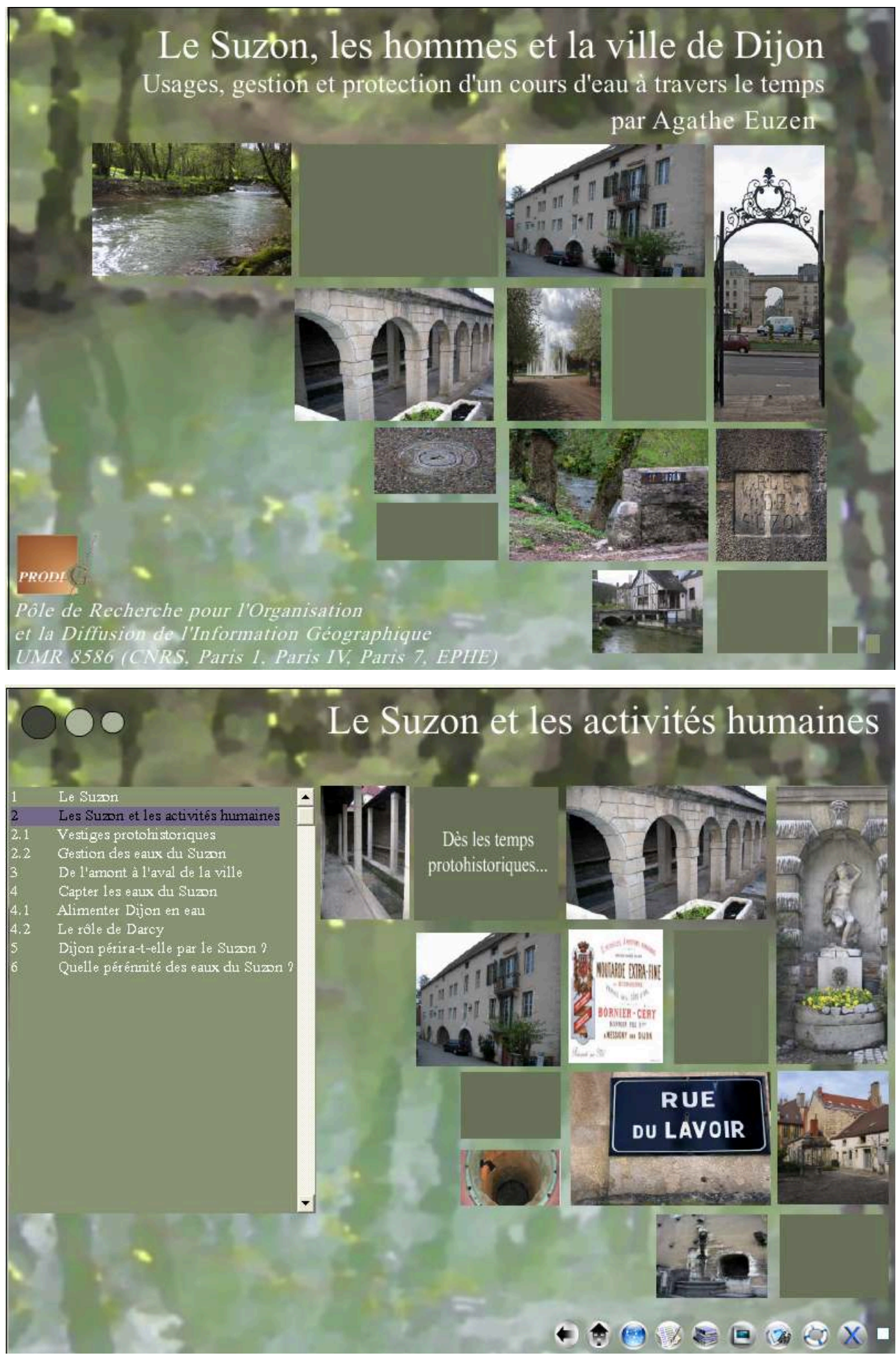

L'arborescence du projet se déplie à volonté grâce à un menu interactif 
7 Grâce à la fonction «synopsis ", le "plan» de la thématique est accessible à tout moment ; ce qui a déjà été affiché se distingue de ce qui n'a pas encore été lu. Le lecteur connaît les chapitres qu'il a déjà abordés et ceux qui attendent sa visite.

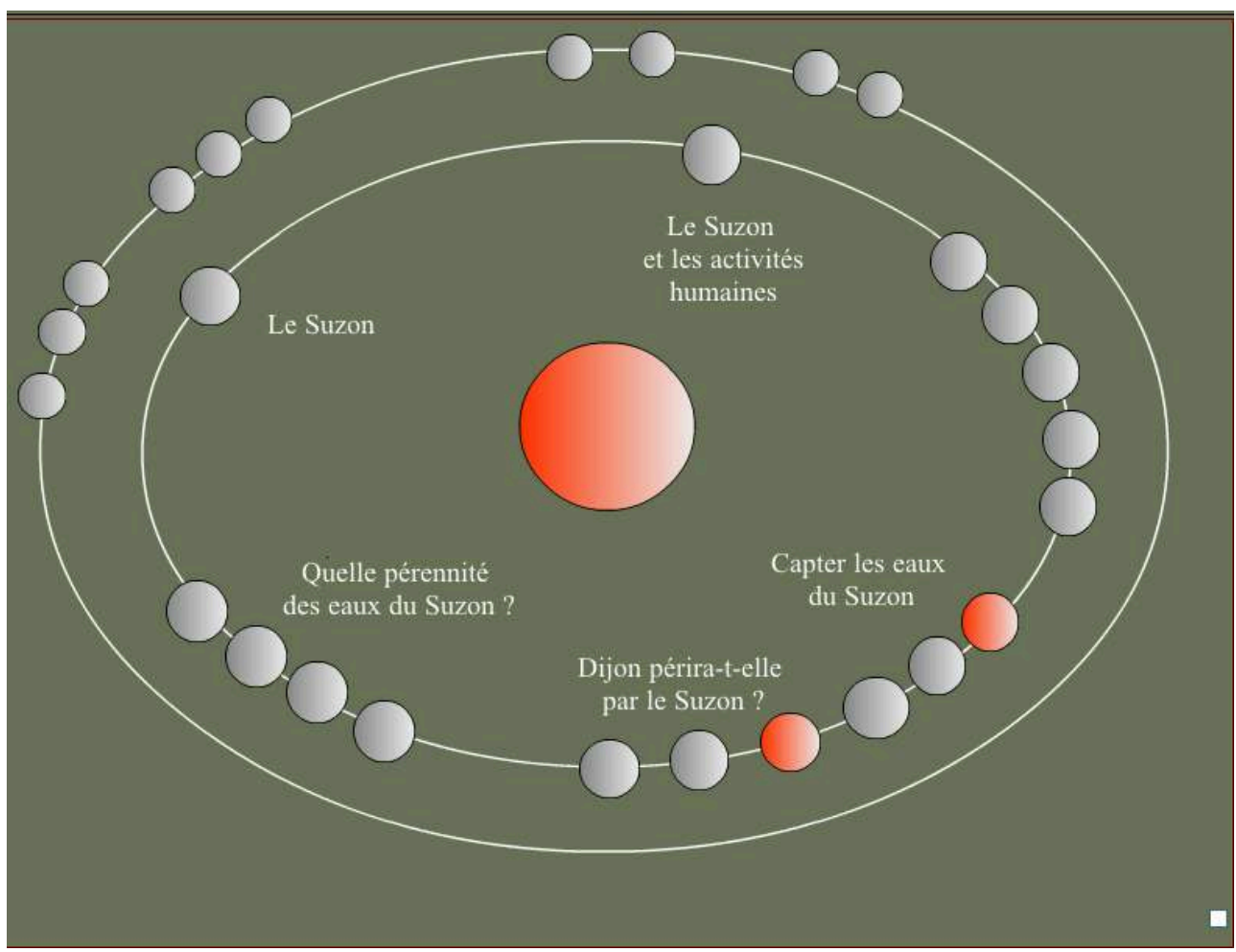

L'ensemble de l'architecture du projet est directement accessible ; les chapitres déjà affichés figurent en rouge

8 La fonction «recherche thématique » permet, à partir d'une liste, de retrouver l'ensemble des médias qui se rattachent à un terme donné. La fonction «glossaire » précise, sous forme de définitions ou de supports graphiques, un ensemble de termes spécifiques à la problématique traitée. 


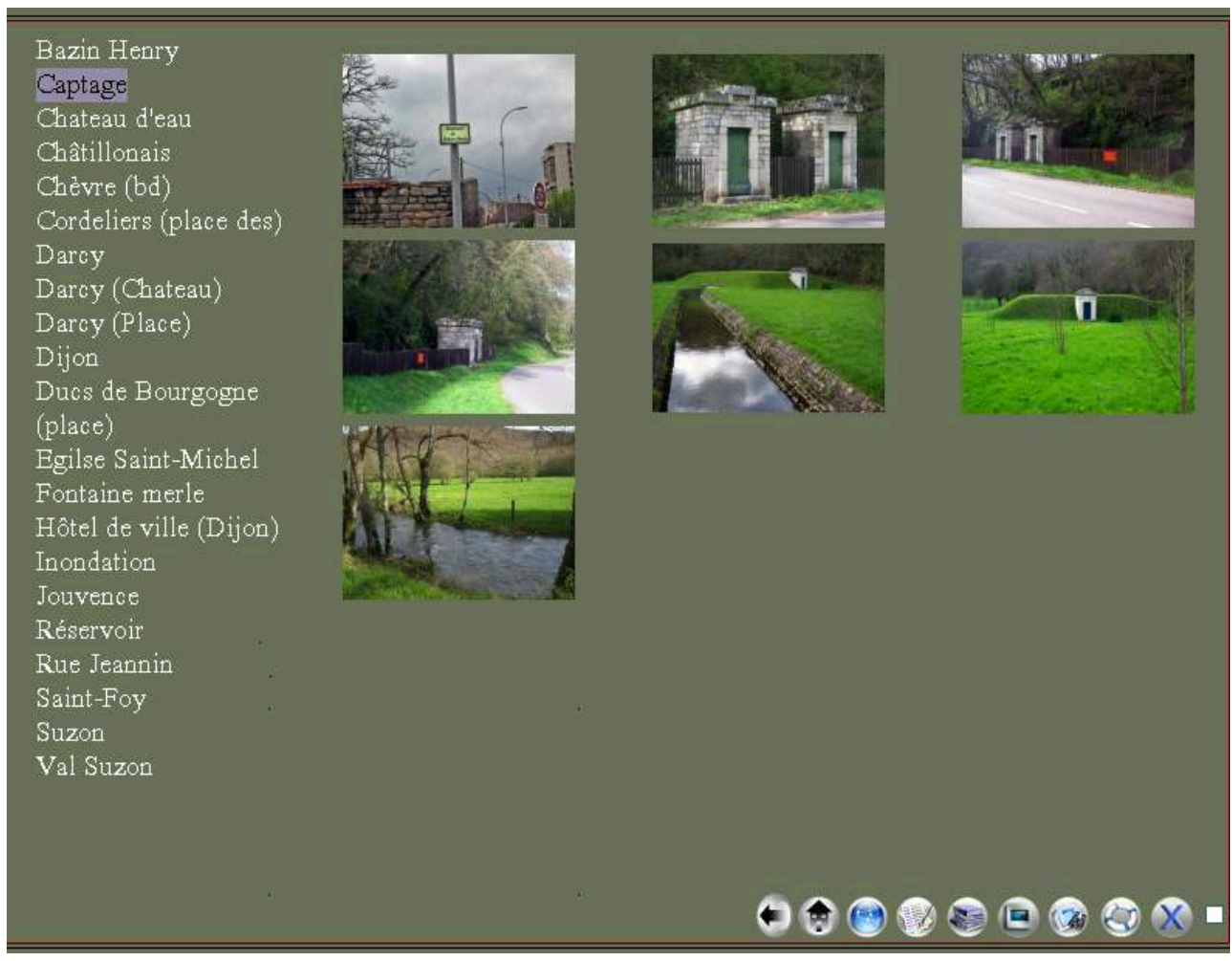

On accède à l'ensemble des médias (photographies, extraits vidéos, cartes...) associés à un terme.

9 Un album photo permet de visualiser sous forme d'un damier l'ensemble des photographies disponibles. Chacune d'entre elles s'affiche sur demande en plein écran.
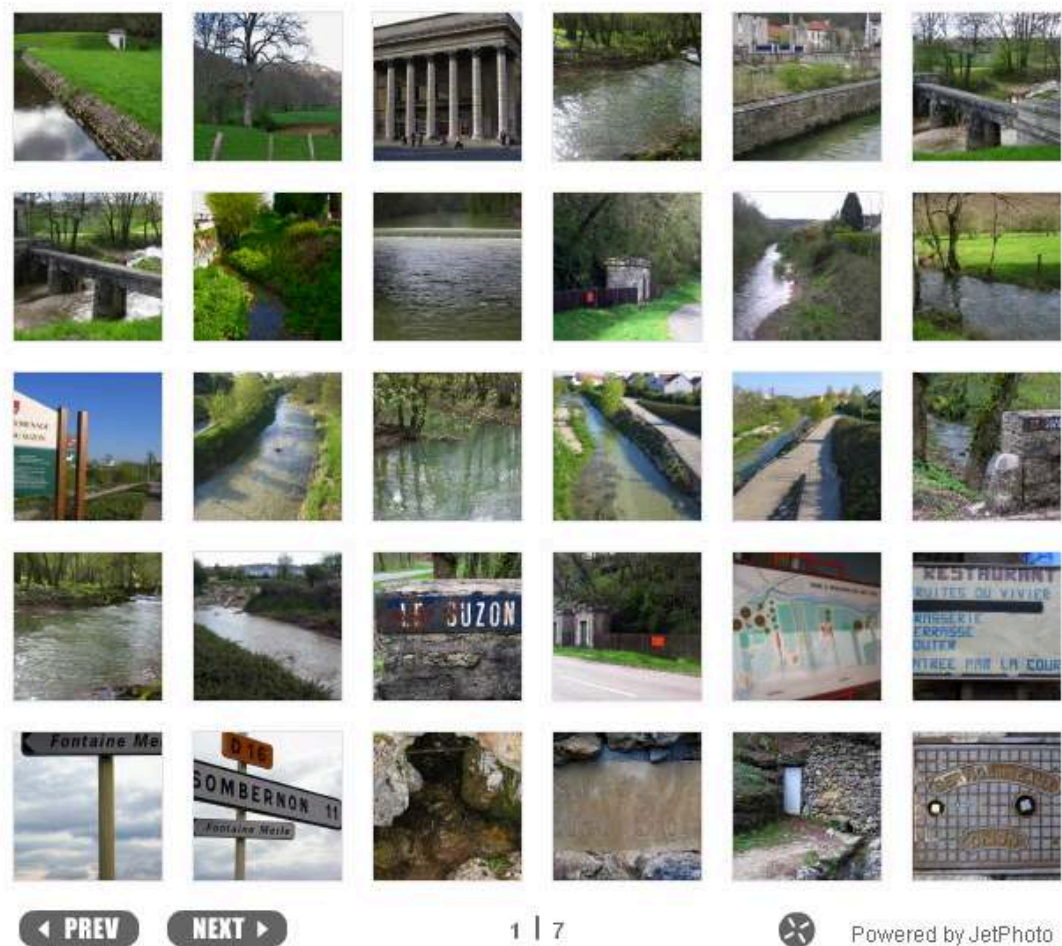

117

(2) Powered by JetPhoto

L'album photos permet de visualiser un nombre important de photographies. 

textes. Ceux-ci doivent impérativement être synthétiques, tant il est vrai qu'un texte long se lit plus facilement sur un support écrit que sur un écran. Là aussi, l'image est au coeur du texte; ce n'est pas une illustration, c'est une contribution à part entière à l'argumentation.

Les termes interactifs figurent en brun ; ils sont enrichis par des images ou par un complément textuel.

11 Les applications multimédias peuvent également proposer deux niveaux de lecture. Le premier est synthétique et immédiatement accessible sous forme de textes courts, de diaporamas ou de vidéos. Le second correspond à un niveau de lecture approfondi ; il propose d'approfondir des thématiques sous forme de textes parfois longs, plutôt destinés à être imprimés (articles, extraits d'ouvrages...).

Ainsi, les applications multimédias offrent une grande richesse fonctionnelle tout en privilégiant l'image fixe ou animée comme messager des thématiques. Elles favorisent aussi une certaine interactivité, laissant par exemple le lecteur choisir son propre itinéraire de lecture. Enfin, elles permettent de tirer profit des développements technologiques: les projets de recherche sont valorisés et diffusés très largement et peut-être à moindre coût grâce aux technologies liées à internet.

13 L'une des difficultés dans la production d'applications multimédias tient bien sûr à la nécessité de disposer d'images fixes ou animées pertinentes d'un point de vue sémantique et technique (bonne résolution, bon format, cadrage correct, images stables....). Un deuxième relève de l'exigence de transmettre des messages synthétiques. Pour laisser sa place à l'image, le texte dit ou écrit doit être le plus court et le plus percutant possible. 


\section{RÉSUMÉS}

La conception et la réalisation d'applications multimédia en géographie privilégient l'image (cartes, films, photographies...) comme messager des thématiques. Elles offrent aussi une grande richesse fonctionnelle et permettent une valorisation et une diffusion large et rapide des principaux résultats de recherche.

The design and the realization of multimedia applications in geography give importance to picture (maps, films, photographs) as a messenger of set of themes. It also provide a large functional richness and allow an utilization and a quick and large diffusion of the principal results of research.

\section{INDEX}

Keywords : image, multimedia, picture, utilization

Mots-clés : valorisation

\section{AUTEUR}

\section{FRANÇOISE DURAFFOUR}

Françoise Duraffour est Ingénieur d'études au CNRS, UMR Prodig. Vous pouvez la joindre : francoise.duraffour@univ-paris1.fr. 